IJMMS 29:7 (2002) 381-388

PII. S0161171202011067

http://ijmms.hindawi.com

(c) Hindawi Publishing Corp.

\title{
PURITY OF THE IDEAL OF CONTINUOUS FUNCTIONS WITH PSEUDOCOMPACT SUPPORT
}

\author{
EMAD A. ABU OSBA
}

Received 7 November 2000 and in revised form 29 March 2001

Let $C_{\Psi}(X)$ be the ideal of functions with pseudocompact support and let $k X$ be the set of all points in $u X$ having compact neighborhoods. We show that $C_{\Psi}(X)$ is pure if and only if $\beta X-k X$ is a round subset of $\beta X, C_{\Psi}(X)$ is a projective $C(X)$-module if and only if $C_{\Psi}(X)$ is pure and $k X$ is paracompact. We also show that if $C_{\Psi}(X)$ is pure, then for each $f \in C_{\Psi}(X)$ the ideal $(f)$ is a projective (flat) $C(X)$-module if and only if $k X$ is basically disconnected ( $F^{\prime}$-space).

2000 Mathematics Subject Classification: 54C30, 54C40, 13C11.

1. Introduction. Let $X$ be a completely regular $T_{1}$-space, $\beta X$ the Stone-Čech compactification of $X$ and $v X$ the Hewitt realcompactification of $X$. Let $C(X)$ be the ring of all continuous real-valued functions defined on $X$. For each $f \in C(X)$, let $Z(f)=\{x \in X: f(x)=0\}, \operatorname{coz} f=X-Z(f)$, the support of $f=S(f)=\mathrm{cl}_{X} \operatorname{coz}(f)$, and $S\left(f^{v}\right)=\operatorname{cl}_{v X} S(f)$, where $f^{v}$ is the extension of $f$ to $u X, S\left(f^{\beta}\right)=\operatorname{cl}_{\beta X} S\left(f^{*}\right)$, where

$$
f^{*}(x)= \begin{cases}1, & f(x) \geq 1, \\ f(x), & -1 \leq f(x) \leq 1, \\ -1, & f(x) \leq-1,\end{cases}
$$

and $f^{\beta}$ is its extension to $\beta X$. If $I$ is an ideal in $C(X)$, then $\operatorname{coz} I=\bigcup_{f \in I} \operatorname{coz} f$.

Let $C_{K}(X), C_{\Psi}(X)$, and $I(X)$ be the ideal of functions with compact support, pseudocompact support, and the intersection of all free maximal ideals of $C(X)$, respectively.

The space $X$ is called $\mu$-compact if $C_{K}(X)=I(X)$, it is called $\Psi$-compact if $C_{K}(X)=$ $C_{\Psi}(X)$, and it is called $\eta$-compact if $C_{\Psi}(X)=I(X)$.

Let $\mu X$ be the smallest $\mu$-compact subspace of $\beta X$ containing $X, \Psi X$ the smallest $\Psi$-compact subspace of $\beta X$ containing $X$, and $\eta X$ the smallest $\eta$-compact subspace of $\beta X$ containing $X$.

The following diagram illustrates the relationships between these spaces:

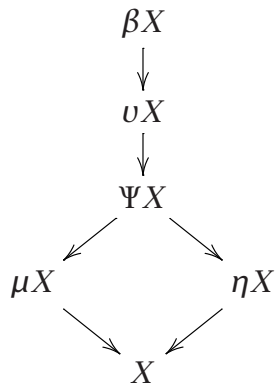


For more information about these spaces the reader may consult [7].

For each subset $A \subseteq \beta X$, let $M^{A}=\left\{f \in C(X): A \subseteq \mathrm{cl}_{\beta X} Z(f)\right\}$ and $O^{A}=\{f \in C(X)$ : $\left.A \subseteq \operatorname{Int}_{\beta X} \mathrm{cl}_{\beta X} Z(f)\right\}=\left\{f \in C(X): A \subseteq \operatorname{Int}_{\beta X} Z\left(f^{\beta}\right)\right\}$. It is well known that $C_{K}(X)=$ $O^{\beta X-X}$ and $C_{\Psi}(X)=O^{\beta X-v X}=M^{\beta X-v X}$. A subset $A$ of $\beta X$ is called a round subset of $\beta X$ if $O^{A}=M^{A}$, see [10].

A space $X$ is called locally pseudocompact if every point of $X$ has a pseudocompact neighborhood (nbhd), it is called basically disconnected if for each $f \in C(X), S(f)$ is clopen in $X$ and it is called an $F^{\prime}$-space if for each $f, g \in C(X)$ such that $f g=0$, then $S(f) \cap S(g)=\varnothing$.

An ideal $I$ of $C(X)$ is called pure if for each $f \in I$, there exists $g \in I$ such that $f=f g$. It is clear that in this case $g=1$ on $S(f)$.

For any undefined terms here the reader may consult [5].

Purity attracted the attention of a lot of people working in ring and module theories. A large class of commutative rings can be classified through the pure ideals of the ring. Purity of some ideals in $C(X)$ was studied by many authors. Kohls [8, Theorem 4.6] called it an ideal with every element having a relative identity. Brookshear [3, page 325] proved that if $X$ is locally compact, then $C_{K}(X)$ is pure, Brookshear [3] and De Marco [4] studied purity and projectivity, Natsheh and Al-Ezeh [11, Theorem 2.4] characterized pure ideals in $C(X)$ to be the ideals of the form $O^{A}$, where $A$ is a unique closed subset of $\beta X$, and Abu Osba and Al-Ezeh [1, Theorem 3.2] proved that $C_{K}(X)$ is pure if and only if $\operatorname{coz} C_{K}(X)=\bigcup_{f \in C_{K}} S(f)$.

In this paper, we characterize purity of $C_{\Psi}(X)$ using the subspace $k X$, the set of all points in $u X$ having compact nbhds, then we use this characterization to study some algebraic properties of this ideal, such as projectivity, when the principal ideal $(f)$ is projective or flat for each $f \in C_{\Psi}(X)$. We found that if $C_{\Psi}(X)$ is pure, then it is projective if and only if $k X$ is paracompact, the principal ideal $(f)$ is projective (flat) if and only if $k X$ is basically disconnected ( $F^{\prime}$-space). An example is given to show that these results are false if $C_{\Psi}(X)$ is not pure.

The following result is well known and is used very often in this article.

Proposition 1.1. For each space $X, C(X)$ is isomorphic to $C(U X)$, and $C_{\Psi}(X)$ is isomorphic to $C_{K}(u X)$.

Proof. Let $\varphi: C(X) \rightarrow C(u X)$ be defined such that $\varphi(f)=f^{u}$. Then $\varphi$ is the required isomorphism, see [5, Section 8.1$]$ and [6, Theorem 2.1].

In this paper, we use the above proposition together with the results we obtained in [1] to characterize purity of the ideal $C_{\Psi}(X)$ using the subspace $k X$.

2. The subspace $k X$. For each ideal $I$ in $C(X)$, define $\theta(I)=\left\{x \in \beta X: I \subseteq M^{x}\right\}$. Then $\theta(I)=\bigcap_{f \in I} \operatorname{cl}_{\beta X} Z(f)$, see [5, Exercise 70.1].

Let $k X=\beta X-\theta\left(C_{\Psi}(X)\right)=\left\{x \in \beta X: C_{\Psi}(X)\right.$ is not contained in $\left.M^{x}\right\}$. The space $k X$ is important in classifying some properties of $X$ and some of its extensions and it is related to the ideal $C_{K}(U X)$. The following propositions and corollaries illustrate this fact. 
Proposition 2.1 (see [6, Corollary 3.3 and Theorems 3.1 and 5.3]). The following statements are equivalent for any space $X$ :

(i) $X$ is locally pseudocompact;

(ii) $X \subseteq k X$;

(iii) $\eta X$ is locally compact;

(iv) $C_{\Psi}(X)$ is not contained in any fixed maximal ideal.

Proposition 2.2 (see [6, Theorems 3.2, 5.1, and 5.2]). For each space $X$,

(i) $k X=\operatorname{Int}_{\beta X} \cup X=\operatorname{Int}_{\beta X} \eta X=\operatorname{Int}_{\beta X} \Psi X$;

(ii) $\eta X=X \cup k X$;

(iii) $\Psi X-X=\bigcup_{f \in C_{\Psi}(X)}\left(S\left(f^{v}\right)-S(f)\right)$.

Proposition 2.3 (see [1, Theorem 2.2]). For each space $X, \operatorname{coz} C_{K}(X)=\operatorname{Int}_{\beta X} X$.

The following result is an easy consequence of Propositions 2.2 and 2.3.

COROLlary 2.4. For each space $X, k X=\operatorname{coz} C_{K}(u X)=\bigcup_{f \in C_{\Psi}(X)} v X-Z\left(f^{v}\right)$.

Corollary 2.5. For each space $X, k X=\bigcup_{f \in C_{\Psi}(X)} \beta X-Z\left(f^{\beta}\right)$.

Proof. Let $f \in C_{\Psi}(X) \subseteq C^{*}(X)$. For each $p \in \beta X-v X, f \in M^{p} \cap C^{*}$.

So $f^{\beta}(p)=0$ for each $p \in \beta X-v X$. Thus $Z\left(f^{\beta}\right)=(\beta X-v X) \cup Z\left(f^{v}\right)$, and $\beta X-$ $Z\left(f^{\beta}\right)=v X \cap\left(\beta X-Z\left(f^{v}\right)\right)=v X-Z\left(f^{v}\right)$.

Now, $\bigcup_{f \in C_{\Psi}(X)} \beta X-Z\left(f^{\beta}\right)=\bigcup_{f \in C_{\Psi}(X)} v X-Z\left(f^{v}\right)=\bigcup_{f^{v} \in C_{K}(v X)} v X-Z\left(f^{v}\right)=k X$, by Corollary 2.4.

THEOREM 2.6. The space $\Psi X$ is locally compact if and only if $X$ is locally pseudocompact and $\theta\left(C_{\Psi}(X)\right)$ is a round subset of $\beta X$.

Proof. See [6, Theorem 5.4] and [10, Theorem 3.3].

3. Purity of $C_{\Psi}(X)$. Here we characterize purity of $C_{\Psi}(X)$ using the subspace $k X$. But first we need some preliminaries.

Proposition 3.1 (see [1, Theorem 3.2]). For each space $X$, the ideal $C_{K}(X)$ is pure if and only if $\operatorname{coz} C_{K}(X)=\bigcup_{f \in C_{K}(X)} S(f)$.

It was proved in [11, Theorem 2.4] that an ideal $I$ in $C(X)$ is pure if and only if $I=O^{A}$ where $A$ is a unique closed subset of $\beta X$. In fact, it was proved that $A$ must be the set $\bigcap_{f \in I} \operatorname{cl}_{\beta X} Z(f)=\theta(I)$. Here we show that if the ideal $O^{A}$ is pure, then $A$ need not be closed, but $O^{A}=O^{\mathrm{cl}_{\beta X} A}$.

THEOREM 3.2. The ideal $O^{A}$ is pure if and only if $O^{A}=O^{\mathrm{cl}_{\beta X}}{ }^{A}$.

Proof. Suppose that $O^{A}$ is pure and $f \in O^{A}$. Then there exists $g \in O^{A}$ such that $f=f g$. So $f^{\beta}=f^{\beta} g^{\beta}$ which implies that $S\left(f^{\beta}\right) \subseteq \operatorname{coz} g^{\beta}$. Hence $A \subseteq \operatorname{Int}_{\beta X} Z\left(g^{\beta}\right) \subseteq$ $Z\left(g^{\beta}\right) \subseteq \beta X-S\left(f^{\beta}\right) \subseteq Z\left(f^{\beta}\right)$. Thus $\mathrm{cl}_{\beta X} A \subseteq Z\left(g^{\beta}\right) \subseteq \beta X-S\left(f^{\beta}\right) \subseteq \operatorname{Int}_{\beta X} Z\left(f^{\beta}\right)$ which implies that $f \in O^{\mathrm{cl}_{\beta X} A}$.

In the following theorem we characterize purity of the ideal $C_{\Psi}(X)$ using properties of the subspace $k X$. 
THEOREM 3.3. The following statements are equivalent:

(1) $C_{\Psi}(X)$ is pure;

(2) $C_{\Psi}(X)=O^{\beta X-k X}$;

(3) $\beta X-k X$ is a round subset of $\beta X$.

Proof. $(1) \Leftrightarrow(2) . C_{\Psi}(X)$ is pure if and only if $C_{\Psi}(X)=O^{\beta X-v X}=O^{\mathrm{cl}_{\beta X}(\beta X-v X)}=$ $O^{\beta X-\operatorname{Int}_{\beta X} \cup X}=O^{\beta X-k X}$, see Proposition 2.2 and Theorem 3.2 above.

(2) $\Rightarrow(3) . M^{\beta X-k X} \supseteq O^{\beta X-k X}=C_{\Psi}(X)=M^{\beta X-u X} \supseteq M^{\beta X-k X}$. So $\beta X-k X$ is a round subset of $\beta X$.

(3) $\Rightarrow(1) . O^{\mathrm{cl}_{\beta X}(\beta X-v X)}=O^{\beta X-\operatorname{Int}_{\beta X} u X}=O^{\beta X-k X}=M^{\beta X-k X}=M^{\mathrm{cl}_{\beta X}(\beta X-v X)}=M^{\beta X-v X}=$ $O^{\beta X-v X}=C_{\Psi}(X)$. So it follows by Theorem 3.2 that $C_{\Psi}(X)$ is pure.

The following result will be extremely useful throughout the rest of the paper.

COROLLARY 3.4. The ideal $C_{\Psi}(X)$ is pure if and only if for each $f \in C_{\Psi}(X), S\left(f^{v}\right) \subseteq k X$.

Proof. The ideal $C_{\Psi}(X)$ is pure if and only if $C_{K}(U X)$ is pure if and only if for each $f \in C_{\Psi}(X), S\left(f^{v}\right) \subseteq k X$, see Propositions 1.1 and 3.1.

COROLlarY 3.5. The space $\Psi X$ is locally compact if and only if $X \subseteq k X$ and $C_{\Psi}(X)$ is pure.

Proof. The result follows from Theorems 2.6 and 3.3.

It was shown in [1, Theorem 3.2] that $C_{K}(X)$ is pure if and only if $\operatorname{coz} C_{K}(X)=$ $\bigcup_{f \in C_{K}(X)} S(f)$. Now, if $C_{\Psi}(X)$ is pure, then it is easy to see that

$$
\operatorname{coz} C_{\Psi}(X)=\bigcup_{f \in C_{\Psi}(X)} S(f) .
$$

This raises the following question: suppose that $\operatorname{coz} C_{\Psi}(X)=\bigcup_{f \in C_{\Psi}(X)} S(f)$, does this imply that $C_{\Psi}(X)$ is pure? The following example shows that this need not be true.

EXAMPLE 3.6. Let $\mathbf{W}^{*}=\left[0, \omega_{1}\right]$ be the set of all ordinals less than or equal to the first uncountable ordinal number $\omega_{1}$. Let $\mathbf{W}=\left[0, \omega_{1}\right)$ and $\mathbf{T}^{*}=\mathbf{W}^{*} \times \mathbb{N}^{*}$, where $\mathbb{N}^{*}$ denote the one point compactification $\mathbb{N} \cup\left\{\omega_{0}\right\}$ of the natural numbers. Let $t=$ $\left(\omega_{1}, \omega_{0}\right), \mathbf{T}=\mathbf{T}^{*}-\{t\}$, let $A=\mathbf{W} \times\left\{\omega_{0}\right\}$ and let $B=\left\{\omega_{1}\right\} \times \mathbb{N}$. Let $\mathbf{S}$ be obtained from $\mathbf{T} \times \mathbb{N}$ by identifying $A \times\{2 n-1\}$ with $A \times\{2 n\}$ and identifying $B \times\{2 n\}$ with $B \times\{2 n+1\}$. Then $\mathbf{S}$ is locally compact, since $\mathbf{T}$ is, and $A \cap B=\varnothing$, see [7, Example 7.3] and [9, page 240]. Let $\mathbf{H}$ be obtained from $\mathbf{T}^{*} \times \mathbb{N}$ by identifying $(A \cup\{t\}) \times\{2 n-1\}$ with $(A \cup\{t\}) \times\{2 n\}$ and identifying $(B \cup\{t\}) \times\{2 n\}$ with $(B \cup\{t\}) \times\{2 n+1\}$. Now, $\mathbf{H}$ is $\sigma$-compact and so it is realcompact. $\mathbf{H}$ is not locally compact since $\left(\omega_{1}, \omega_{0}, n\right)$ has no compact neighborhood for each $n \in \mathbb{N}$. So $\mathbf{S} \subseteq k \mathbf{S} \subseteq u \mathbf{S} \subseteq \mathbf{H}$. Define $f: \mathbf{S} \rightarrow \mathbb{R}$ by $f(\alpha, n, 1)=1 / n$ for all $\alpha \in \mathbf{W}^{*}, n \in \mathbb{N}$ and by zero otherwise. Then coz $f=\mathbf{W}^{*} \times$ $\mathbb{N} \times\{1\}$ and $S(f)=\mathbf{T} \times\{1\}$ is pseudocompact, noncompact. So $f \in C_{\Psi}(\mathbf{S})-C_{K}(\mathbf{S})$, which implies that $\mathbf{S}$ is not $\Psi$-compact. Hence $k \mathbf{S}=\mathbf{S} \neq \Psi \mathbf{S}$. Therefore, $\Psi \mathbf{S}$ is not locally compact. So it follows by Corollary 3.5 that $C_{\Psi}(\mathbf{S})$ is not pure although $\mathbf{S}$ is locally pseudocompact and $\operatorname{coz} C_{\Psi}(\mathbf{S})=\mathbf{S}=\bigcup_{f \in C_{\Psi}} S(f)$.

4. Some applications. In this section, we use the characterization obtained in Theorem 3.3 and Corollary 3.4 above for purity of the ideal $C_{\Psi}(X)$ to characterize 
when $C_{\Psi}(X)$ is a projective $C(X)$-module, when every principal ideal of $C_{\Psi}(X)$ is projective or flat $C(X)$-module, and for which spaces $X$ and $Y$, the two ideals $C_{\Psi}(X)$ and $C_{\Psi}(Y)$ are isomorphic.

THEOREM 4.1. Let $C_{\Psi}(X)$ and $C_{\Psi}(Y)$ be pure ideals. Then $C_{\Psi}(X)$ is isomorphic to $C_{\Psi}(Y)$ if and only if $k X$ is homeomorphic to $k Y$.

Proof. If $C_{\Psi}(X)$ is isomorphic to $C_{\Psi}(Y)$, then $k X$ is homeomorphic to $k Y$, see [12, Corollary 4.11].

For the converse, we prove that $C_{K}(v X)$ is isomorphic to $C_{K}(v Y)$, then the result follows from Proposition 1.1.

Suppose $\varphi: k X \rightarrow k Y$ is a homeomorphism. Let $f \in C_{K}(u Y)$, then $f_{1} \circ \varphi \in C(k X)$, where $f_{1}=f_{\left.\right|_{k Y}}$. But coz $f=\varphi\left(\operatorname{coz}\left(f_{1} \circ \varphi\right)\right)$, which implies that $\varphi^{-1}(\operatorname{coz} f)=\operatorname{coz}\left(f_{1} \circ \varphi\right)$.

Therefore $\mathrm{cl}_{k X} \operatorname{coz}\left(f_{1} \circ \varphi\right)=\mathrm{cl}_{k X} \varphi^{-1}(\operatorname{coz} f)=\varphi^{-1}(S(f))$, since $S(f)$ is contained in $k Y$ by the purity of $C_{\Psi}(Y)$. Now, for each $f \in C_{K}(u X)$ define

$$
g_{f}: u X \rightarrow \mathbb{R} \text { by } g_{f}(x)= \begin{cases}f_{1} \circ \varphi(x), & x \in k X, \\ 0, & x \in v X-\varphi^{-1}(S(f)) .\end{cases}
$$

Then, $g_{f} \in C_{K}(u X)$, since $S\left(g_{f}\right)=\mathrm{cl}_{k X} \operatorname{coz}\left(f_{1} \circ \varphi\right)$ is compact.

Define $\bar{\varphi}: C_{K}(u Y) \rightarrow C_{K}(u X)$ by $\bar{\varphi}(f)=g_{f}$. Then $\bar{\varphi}$ is a ring homomorphism. It remains to show that $\bar{\varphi}$ is bijective.

To see that $\bar{\varphi}$ is one-to-one, suppose $\bar{\varphi}(f)=0$. Then $f_{1} \circ \varphi(x)=0$ for every $x \in k X$. But $\operatorname{coz}\left(f_{1} \circ \varphi\right)=\varphi^{-1}(\operatorname{coz} f)$, and so $\varphi^{-1}(\operatorname{coz} f)=\varnothing$. Therefore $f=0$.

To see that $\bar{\varphi}$ is onto, let $f \in C_{K}(u X)$. Define

$$
g: v Y \rightarrow \mathbb{R} \quad \text { by } g(y)= \begin{cases}f \circ \varphi^{-1}(y), & y \in k Y, \\ 0, & y \in v Y-\varphi(S(f)) .\end{cases}
$$

Then $g \in C(v Y)$, since $\varphi(S(f))$ is compact. Here again we use the purity of $C_{\Psi}(X)$, since we assumed that $S(f) \subseteq k X$. Moreover, if $g(y) \neq 0$, then $\varphi^{-1}(y) \in \operatorname{coz} f$. So $\operatorname{coz} g \subseteq \varphi(\operatorname{coz} f)$.

Thus, $\mathrm{cl}_{k Y} \operatorname{coz} g \subseteq \mathrm{cl}_{k Y} \varphi(\operatorname{coz} f)=\varphi\left(\mathrm{cl}_{U X} \operatorname{coz} f\right)=\varphi(S(f))$. Hence $S(g)=\mathrm{cl}_{k Y} \operatorname{coz} g$ is compact. It follows that $g \in C_{K}(u Y)$.

Finally, note that

$$
\begin{aligned}
\bar{\varphi}(g)(x) & = \begin{cases}g_{1} \circ \varphi(x), & x \in k X, \\
0, & x \in v X-\varphi^{-1}(S(g)) ;\end{cases} \\
& = \begin{cases}f \circ \varphi^{-1} \circ \varphi(x), & x \in k X, \\
0, & x \in v X-\varphi^{-1}(S(g)) ;\end{cases} \\
& = \begin{cases}f(x), & x \in k X, \\
0, & \text { otherwise }\end{cases} \\
& =f(x) .
\end{aligned}
$$

Thus $\bar{\varphi}(g)=f$ and so $\bar{\varphi}$ is onto. Hence $C_{K}(u X)$ is isomorphic to $C_{K}(u Y)$. 
Here we characterize when $C_{\Psi}(X)$ is a projective $C(X)$-module.

THEOREM 4.2. The ideal $C_{\Psi}(X)$ is a projective $C(X)$-module if and only if $k X$ is paracompact and $C_{\Psi}(X)$ is pure.

Proof. It was proved by Brookshear [3, Theorem 3.10] that $C_{K}(X)$ is a projective $C(X)$-module if and only if $\operatorname{coz} C_{K}(X)$ is paracompact and $S(f) \subseteq \operatorname{coz} C_{K}(X)$ for each $f \in C_{K}(X)$. Our result now follows from Proposition 3.1 and Corollaries 2.4 and 3.4.

It was proved in [2, Lemma 2] and [3, Corollary 2.5] that the principal ideal $(f)$ is a projective (flat) $C(X)$-module if and only if $S(f)$ is clopen in $X(\operatorname{Ann}(f)$ is pure). We can use this result to determine when the principal ideal $(f)$ is a projective or a flat $C(X)$-module for each $f \in C_{\Psi}(X)$.

THEOREM 4.3. For each $f \in C_{\Psi}(X)$, the ideal $(f)$ is a projective $C(X)$-module if and only if $C_{\Psi}(X)$ is pure and $k X$ is basically disconnected.

Proof. Suppose that $k X$ is basically disconnected and $C_{\Psi}(X)$ is pure. Let $f \in$ $C_{\Psi}(X)$. Then $S\left(f^{v}\right) \subseteq k X$ since $C_{\Psi}(X)$ is pure. Now let $f_{1}=f^{v}{ }_{\mid k X}$ and note that $\mathrm{cl}_{k X}\left(k X-Z\left(f_{1}\right)\right)=S\left(f^{v}\right)$. Since $k X$ is basically disconnected, $S\left(f^{v}\right)$ is open in $k X$ and therefore it is open in $u X$ (cf. Proposition 2.2). Thus $S(f)=S\left(f^{v}\right) \cap X$ is open in $X$. Hence the ideal $(f)$ is a projective $C(X)$-module.

Conversely, suppose that every principal ideal of $C_{\Psi}(X)$ is a projective $C(X)$-module. For each $f \in C_{\Psi}(X), S(f)$ is open in $X$, so define

$$
g(x)= \begin{cases}1, & x \in S(f), \\ 0, & \text { otherwise }\end{cases}
$$

Then $g \in C_{\Psi}(X)$ and $f=f g$. Thus $C_{\Psi}(X)$ is a pure ideal.

To demonstrate basic disconnectedness, we first show that for each $f \in C_{K}(k X)$, $S(f)$ is clopen. Then we will use this result to show that for each $k \in C(k X), S(k)$ is clopen.

Let $f \in C_{K}(k X)$. Then $f$ can be extended to a function $F \in C_{K}(u X)$ with $\mathrm{cl}_{k X}(k X-$ $Z(f))=S(F)$ which is open, since $C_{\Psi}(X)$ is isomorphic to $C_{K}(u X)$.

Now, let $k \in C(k X)$, and $a \in \mathrm{cl}_{k X}(k X-Z(k)) \subseteq k X$. So there exists an open set $U$ such that $\bar{U}$ is compact, and $a \in U \subseteq \bar{U} \subseteq k X$. There exists $f \in C(k X)$ such that $f(a)=1$ and $f(k X-U)=0$. Then $f \in C_{K}(k X)$.

Thus $a \in(k X-Z(f)) \cap \mathrm{cl}_{k X}(k X-Z(k)) \subseteq \mathrm{cl}_{k X}\left((k X-Z(f)) \cap \mathrm{cl}_{k X}(k X-Z(k))\right)=$ $\mathrm{cl}_{k X}((k X-Z(f)) \cap(k X-Z(k)))=\mathrm{cl}_{k X}(k X-Z(f k)) \subseteq \mathrm{cl}_{k X}(k X-Z(k))$. But $\mathrm{cl}_{k X}(k X-$ $Z(f k))$ is compact, and so is clopen since $f k \in C_{K}(k X)$. So, $\mathrm{cl}_{k X}(k X-Z(k))$ is clopen in $k X$. Thus $k X$ is basically disconnected.

THEOREM 4.4. Let $X$ be a space such that $C_{\Psi}(X)$ is pure. Then for each $f \in C_{\Psi}(X)$ the principal ideal $(f)$ is a flat $C(X)$-module if and only if $k X$ is an $F^{\prime}$-space.

Proof. Suppose that $k X$ is an $F^{\prime}$-space, $f \in C_{\Psi}(X)$ and $g \in \operatorname{Ann}(f)$. Let $f_{1}=f^{v}{ }_{\mid k X}$ and $g_{1}=g^{v}{ }_{k X}$. Then $\left(k X-Z\left(f_{1}\right)\right) \cap\left(k X-Z\left(g_{1}\right)\right)=\varnothing$. So, $\mathrm{cl}_{k X}\left(k X-Z\left(f_{1}\right)\right) \cap \mathrm{cl}_{k X}(k X-$ $\left.Z\left(g_{1}\right)\right)=\varnothing$, since $k X$ is an $F^{\prime}$-space. But $S\left(f^{v}\right)=\operatorname{cl}_{k X}\left(k X-Z\left(f_{1}\right)\right)$, since $C_{\Psi}(X)$ is 
pure and $S\left(f^{v}\right) \subseteq k X$. Now if $x \in S\left(f^{v}\right)$, then $x \in \mathrm{cl}_{k X}\left(k X-Z\left(f_{1}\right)\right)$, which implies that $x \notin \mathrm{cl}_{k X}\left(k X-Z\left(g_{1}\right)\right)$. So there exists an open set $U \subseteq k X$ such that $x \in U$ and $U \cap\left(k X-Z\left(g_{1}\right)\right)=\varnothing$. Therefore $x \in U \subseteq Z\left(g_{1}\right) \subseteq Z\left(g^{v}\right)$. But $U$ is open in $u X$, since $k X$ is. So $U \cap\left(u X-Z\left(g^{v}\right)\right)=\varnothing$, which implies that $x \notin S\left(g^{v}\right)$. Thus $S\left(f^{v}\right) \cap S\left(g^{v}\right)=\varnothing$.

The compactness of $S\left(f^{v}\right)$ implies that there exists $k^{v} \in C(v X)$ such that $k^{v}\left(S\left(f^{v}\right)\right)$ $=0$ and $k^{v}\left(S\left(g^{v}\right)\right)=1$. So, $k \in \operatorname{Ann}(f)$, and $g=g k$. Thus the ideal $(f)$ is a flat $C(X)$ module since $\operatorname{Ann}(f)$ is pure.

Conversely, suppose that the principal ideal $(f)$ is a flat $C(X)$-module for each $f \in$ $C_{\Psi}(X)$. Let $g, k \in C(k X)$ such that $g k=0$. Suppose $y \in \mathrm{cl}_{k X}(k X-Z(g)) \cap \mathrm{cl}_{k X}(k X-$ $Z(k))$. There exists $f_{1} \in C_{K}(u X)$ such that $f_{1}(y) \neq 0$. Let $f=\left.f_{1}\right|_{k X}$, then $y \in$ $\mathrm{cl}_{k X}(k X-Z(f g)) \cap \mathrm{cl}_{k X}(k X-Z(f k))$. Define

$$
\begin{aligned}
& h_{1}(x)= \begin{cases}f g(x), & x \in \mathrm{cl}_{k X}(k X-Z(f g)), \\
0, & x \in v X-(k X-Z(f g)) ;\end{cases} \\
& h_{2}(x)= \begin{cases}f k(x), & x \in \mathrm{cl}_{k X}(k X-Z(f k)), \\
0, & x \in v X-(k X-Z(f k)) .\end{cases}
\end{aligned}
$$

Then $h_{1}, h_{2} \in C_{K}(u X)$, since $S\left(h_{1}\right)$ and $S\left(h_{2}\right)$ are compact sets. Moreover, $h_{1} h_{2}=0$. So, there exists $h_{1}^{\prime} \in \operatorname{Ann}\left(h_{2}\right)$ such that $h_{1}=h_{1} h_{1}^{\prime}$. Hence $y \in \operatorname{cl}_{k X}(k X-Z(f g))=$ $S\left(h_{1}\right) \subseteq \operatorname{coz} h_{1}^{\prime}$. But $h_{1}^{\prime}\left(S\left(h_{2}\right)\right)=0$, so $y \notin S\left(h_{2}\right)=\mathrm{cl}_{k X}(k X-Z(f k))$, a contradiction. Hence $\operatorname{cl}_{k X}(k X-Z(g)) \cap \mathrm{cl}_{k X}(k X-Z(k))=\varnothing$ and $k X$ is an $F^{\prime}$-space.

EXAMPLE 4.5. Let $X=[-1,1]$ with all its points isolated, except for $x=0$ it has its usual nbhds. Then $X$ is regular, paracompact, and consequently realcompact. So $X=u X$ and $k X=X-\{0\} \subseteq X$.

Let

$$
f(x)= \begin{cases}x, & x=\frac{1}{n}, n \in \mathbb{Z}^{*} \\ 0, & \text { otherwise. }\end{cases}
$$

Then $S(f)=\left\{1 / n: n \in \mathbb{Z}^{*}\right\} \cup\{0\}$. So $f \in C_{\Psi}(X)$ and $S(f)$ is not contained in $k X$. So $C_{\Psi}(X)$ is not a pure ideal.

The set $S(f)$ is not open, so the ideal $(f)$ is not projective. Ann $(f)$ is not pure, since the function

$$
g(x)= \begin{cases}0, & x=\frac{1}{n}, n \in \mathbb{Z}^{*} \\ x, & \text { otherwise }\end{cases}
$$

belongs to $\operatorname{Ann}(f)$, but $S(g)=X-\left\{1 / n: n \in \mathbb{Z}^{*}\right\}$ is not a subset of $\operatorname{coz} \operatorname{Ann}(f)$, since for each $h \in \operatorname{Ann}(f), h(0)=0$. So the ideal $(f)$ is not a flat $C(X)$-module. Let $Y=X-\{0\}$, then $k X=Y=k Y$, but $C_{\Psi}(X)$ is not isomorphic to $C_{\Psi}(Y)$, since the latter is pure.

This example shows that Theorems $4.1,4.2,4.3$, and 4.4 need not be true if $C_{\Psi}(X)$ is not pure. 


\section{REFERENCES}

[1] E. A. Abu Osba and H. Al-Ezeh, Purity of the ideal of continuous functions with compact support, Math. J. Okayama Univ. 41 (1999), 111-120.

[2] H. Al-Ezeh, M. A. Natsheh, and D. Hussein, Some properties of the ring of continuous functions, Arch. Math. (Basel) 51 (1988), no. 1, 60-64.

[3] J. G. Brookshear, Projective ideals in rings of continuous functions, Pacific J. Math. 71 (1977), no. 2, 313-333.

[4] G. De Marco, Projectivity of pure ideals, Rend. Sem. Mat. Univ. Padova 69 (1983), 289-304.

[5] L. Gillman and M. Jerison, Rings of Continuous Functions, Graduate Texts in Mathematics, no. 43, Springer-Verlag, New York, 1976.

[6] D. Harris, The local compactness of $v X$, Pacific J. Math. 50 (1974), 469-476.

[7] D. G. Johnson and M. Mandelker, Functions with pseudocompact support, General Topology and Appl. 3 (1973), 331-338.

[8] C. W. Kohls, Ideals in rings of continuous functions, Fund. Math. 45 (1957), 28-50.

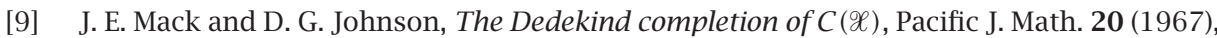
231-243.

[10] M. Mandelker, Round $z$-filters and round subsets of $\beta X$, Israel J. Math. 7 (1969), 1-8.

[11] M. A. Natsheh and H. Al-Ezeh, Characterization of pure ideals in $C(X)$, J. Fac. Sci. UAE Univ. 3 (1991), no. 1, 17-23.

[12] D. Rudd, On isomorphisms between ideals in rings of continuous functions, Trans. Amer. Math. Soc. 159 (1971), 335-353.

Emad A. Abu Osba: Department of Mathematics, University of Petra, Amman 961343, JORDAN

E-mail address: emad@uop.edu.jo 


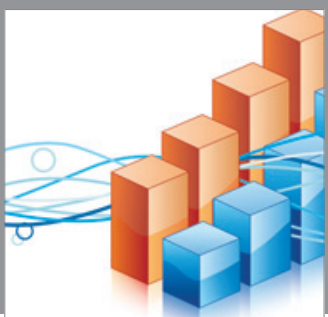

Advances in

Operations Research

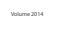

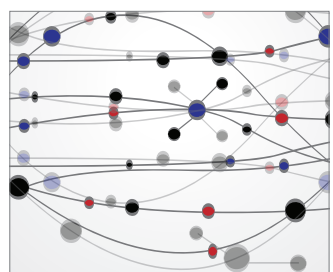

\section{The Scientific} World Journal
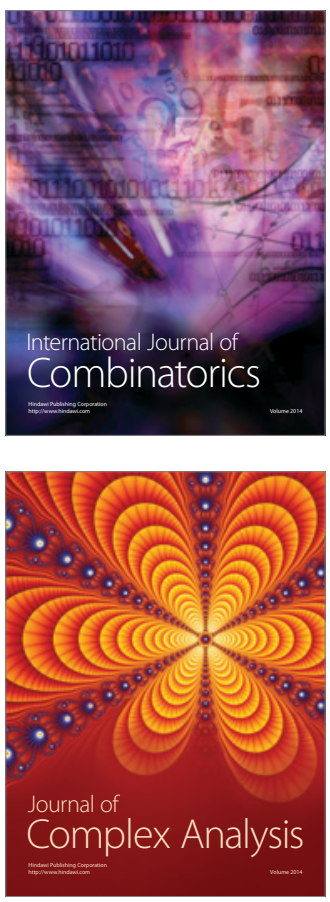

International Journal of

Mathematics and

Mathematical

Sciences
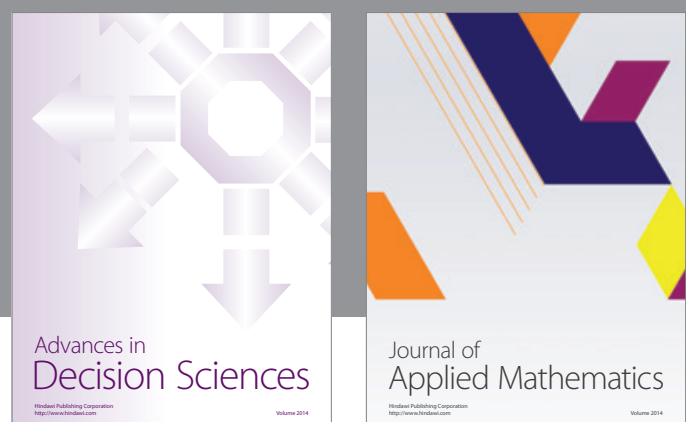

Journal of

Applied Mathematics
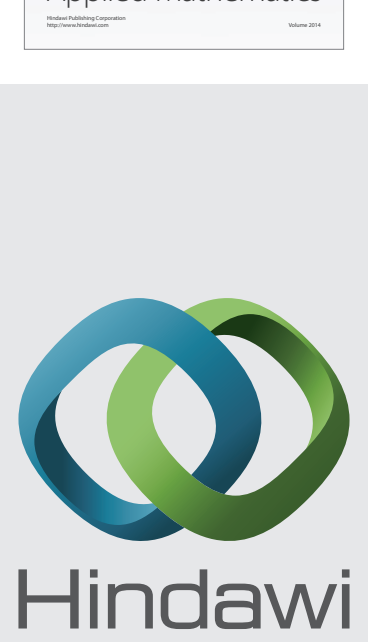

Submit your manuscripts at http://www.hindawi.com
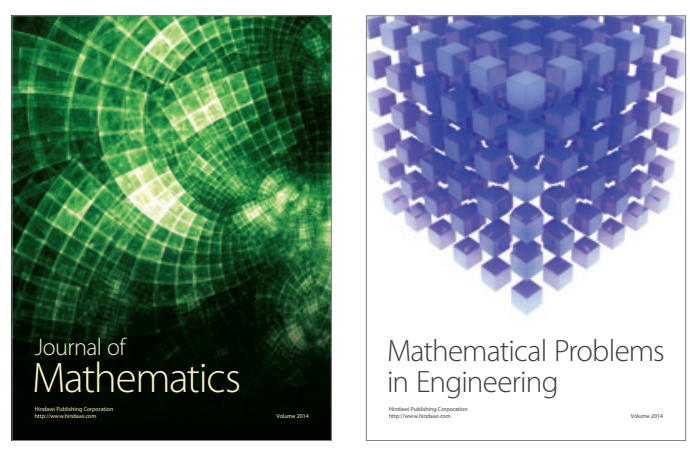

Mathematical Problems in Engineering
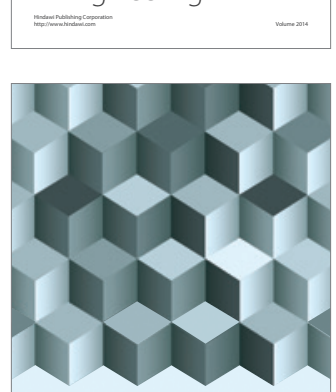

Journal of

Function Spaces
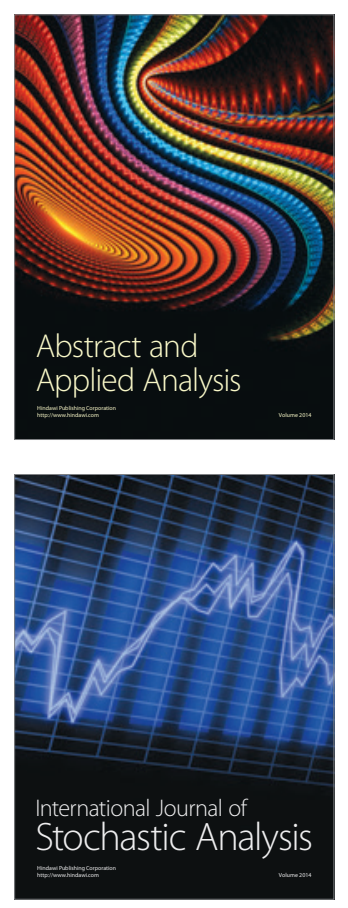

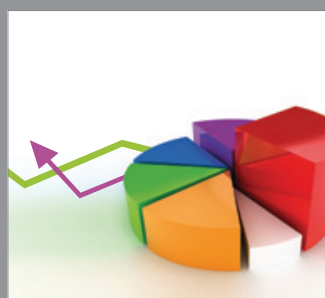

ournal of

Probability and Statistics

Promensencen
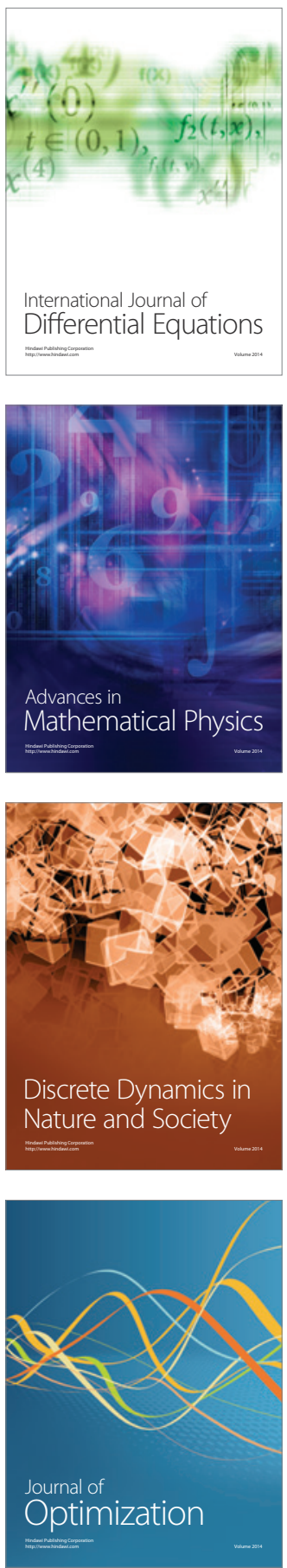